\title{
Phase differences in reaction-diffusion-advection systems and applications to morphogenesis
}

\author{
Abbey J. Perumpanan, Jonathan A. Sherratt and Philip K. Main \\ Mathematical Institute, 24-29 St. Giles', Oxford OX1 3LB, UK
}

[Received 28 November 1994 and in revised form 18 February 1995]

\begin{abstract}
The authors study the effect of advection on reaction-diffusion patterns. It is shown that the addition of advection to a two-variable reaction-diffusion system with periodic boundary conditions results in the appearance of a phase difference between the patterns of the two variables which depends on the difference between the advection coefficients. The spatial patterns move like a travelling wave with a fixed velocity which depends on the sum of the advection coefficients. By a suitable choice of advection coefficients, the solution can be made stationary in time. In the presence of advection a continuous change in the diffusion coefficients can result in two Turing-type bifurcations as the diffusion ratio is varied, and such a bifurcation can occur even when the inhibitor species does not diffuse. It is also shown that the initial mode of bifurcation for a given domain size depends on both the advection and diffusion coefficients. These phenomena are demonstrated in the numerical solution of a particular reaction-diffusion system, and finally a possible application of the results to pattern formation in Drosophila larvae is discussed.
\end{abstract}

\section{Introduction}

Diffusion driven instability was first proposed as a mechanism of developmental pattern formation by Turing (1952). In this mechanism reacting and diffusing chemicals (morphogens) form a pattern which controls subsequent cell differentiation. Subsequent research has led to a detailed understanding of the mechanism and its applications (see Murray, 1989, and Meinhardt, 1982, for a review) and many variants have been studied (e.g. Benson et al., 1993; Dillon et al. 1994). However, one feature common to all variants is that the prepattern of the different morphogens is either in phase or wholly out of phase. This limits the applicability of diffusion-driven instability: in particular Russell (1985) has shown that a chemical prepattern in which the morphogens have inexact phase differences could be used to explain metamerism (segmentation) and its mutant variants in the Drosophila larva. In this paper we discuss reaction-diffusionadvection systems as possible candidates for the generation of chemical waves with inexact phase differences.

† Present address: Nonlinear Systems Laboratory, Mathematics Institute, University of Warwick, Coventry CV4 7AZ. 


\section{Derivation of the model}

Consider a system of reacting morphogens, say $u=\left(u_{1}, u_{2}, \ldots, u_{n}\right)$, each of which exhibits advection in addition to diffusing through the surrounding medium. In order to describe their behaviour, a continuum approach using density functions to describe the distribution of the basic particles is typically used. For the $i$ th chemical morphogen we define $u_{i}(x, t)$ as the concentration, $Q_{l}(\boldsymbol{x}, t)$ as the net creation rate of particles, and $J_{l}(\boldsymbol{x}, t)$ as the flux density in the region $\Omega$. The conservation equations for these chemicals have the form

$$
\frac{\partial u_{i}}{\partial t}=-\nabla \cdot J+Q
$$

In our model we assume $J_{i}=-d_{i} \nabla u_{i}+a_{i} u_{i}$, where $a_{i}$ and $d_{i}$ are constants. This implies that the flux depends both on the concentration (advection) and the gradient (diffusion). Hence we have the system of equations for the $n$ morphogens of the form

$$
\boldsymbol{u}_{t}=\boldsymbol{D} \cdot \nabla^{2} \boldsymbol{u}+\nabla(\boldsymbol{a} \cdot \boldsymbol{u})+f(\boldsymbol{u})
$$

We consider for simplicity the case of two interacting morphogens $u$ and $v$, in one space dimension, so that

$$
\begin{aligned}
& \frac{\partial u}{\partial t}=\gamma f(u, v)+a \frac{\partial u}{\partial x}+D_{u} \frac{\partial^{2} u}{\partial x^{2}} \\
& \frac{\partial v}{\partial t}=\gamma g(u, v)+b \frac{\partial v}{\partial x}+D_{v} \frac{\partial^{2} v}{\partial x^{2}}
\end{aligned}
$$

for $0<x<1$. Here $\gamma$ is a scale parameter, resulting from the rescaling of the domain. We assume that the kinetics $f$ and $g$ are such that there is a homogeneous steady state, $\left(u_{0}, v_{0}\right)$ say. For algebraic simplicity, we will in future write $p=a+b$ and $q=a-b$.

We now consider the most appropriate boundary conditions for (3) and (4). Most reaction-diffusion models for pattern formation have used zero-flux boundary conditions mainly because the interest is in self-organization of pattern, and zero flux implies no external input. However, it would be artificial to study phase differences with either zero flux or Dirichlet boundary conditions, since these impose a phase difference on any solution, at least near the boundaries. In fact, in the context of developmental biology we are in most cases studying a domain which is much larger than the size of the pattern, since the pattern is localized to one part of the embryo. In such cases, we do not expect the boundary conditions to have any significant bearing on the pattern formed. Hence the pattern we are studying should be driven by the equations and not by the boundary conditions. In some contexts it is appropriate to consider an infinite domain, but in others the restrictions on pattern imposed by a finite domain size are crucial, and in such cases periodic boundary conditions represent a convenient way of studying patterning excluding effects from the boundary, except the 
constraint of discrete mode numbers. Periodic boundary conditions correspond to closed domains and were in fact used by Turing (1952) in his original paper. The conditions are

$$
\begin{array}{ll}
u(0)=u(1), & u^{\prime}(0)=u^{\prime}(1), \\
v(0)=v(1), & v^{\prime}(0)=v^{\prime}(1) .
\end{array}
$$

The ensuing analysis is equally valid for an infinite domain; the imposition of (5) and (6) corresponds to mode selection, which will be discussed in detail later.

The homogeneous steady state $\left(u_{0}, v_{0}\right)$ is the positive solution of

$$
f(u, v)=0, \quad g(u, v)=0 .
$$

We are looking for instability that is prompted by the spatial spread of the morphogen, and hence we require the steady state to be linearly stable to spatially homogeneous perturbations, for which the conditions are

$$
\begin{aligned}
f_{u}+g_{v} & <0, \\
f_{u} g_{v}-f_{u} g_{u} & >0
\end{aligned}
$$

(see e.g. Murray, 1989). These impose certain constraints on the kinetic parameters. Now we consider the full reaction-diffusion-advection system and linearize about the homogeneous steady state, giving

$$
w_{t}=\gamma A w+B w_{x}+D w_{x x},
$$

where

$$
\boldsymbol{B}=\left[\begin{array}{ll}
a & 0 \\
0 & b
\end{array}\right], \quad \boldsymbol{D}=\left[\begin{array}{ll}
1 & 0 \\
0 & d
\end{array}\right]
$$

and $\boldsymbol{w}$ is the vector of perturbations from the steady state. We look for solutions of the form

$$
\boldsymbol{w}=\boldsymbol{w}_{0} \mathrm{e}^{\lambda} \boldsymbol{W}(x)
$$

Since we are considering either an infinite domain or a finite domain with periodic boundary conditions, any solution of the linear equation (10) can be written as a sum of sinusoidal solutions of the form

$$
W(x)=c_{1} \mathrm{e}^{\mathrm{i} k x}+c_{2} \mathrm{e}^{-\mathrm{ikx}} .
$$

The linearized equation determines a growth rate $\lambda$ corresponding to each admissible wavenumber $k$. The steady state will be unstable to suitable perturbations if any of these growth rates are positive, while if all are negative the steady state will be stable. The inherent stochasticity in all biological systems means that in applications all wavenumbers will be present in perturbations. It is noteworthy that other approaches have been used in the past for studying the stability properties of solutions to reaction-diffusion equations. For example, Casten \& Holland (1977) have described a method to determine the local asymptotic stability of a nonlinear reaction-diffusion system with zero Neumann boundary 
conditions based on the eigenvalues of a matrix arising from the linearization of the system of equations.

\section{Conditions for dispersion driven instability}

Substituting (11) into (3) and (4) gives

$$
\begin{aligned}
& \lambda u_{0}=-k^{2} u_{0}+a i k u_{0}+\gamma\left(f_{u} u_{0}+f_{v} v_{0}\right), \\
& \lambda v_{0}=-d k^{2} v_{0}+b i k v_{0}+\gamma\left(g_{u} u_{0}+g_{v} v_{0}\right) .
\end{aligned}
$$

This has a nontrivial solution if and only if

$$
\operatorname{det}\left|\lambda I-\gamma A-B \mathrm{i} k+D k^{2}\right|=0 \text {. }
$$

This equation is the dispersion relation, determining the growth rate $\lambda$ as a function of the wavenumber $k$. Expanding it gives

where

$$
\lambda^{2}+\lambda\left[k^{2}(1+d)-i k(a+b)-\gamma\left(f_{u}+g_{v}\right)\right]+h(k)=0,
$$

$h(k)=d k^{4}-i k^{3}(a d+b)-k^{2}\left[\gamma\left(d f_{u}+g_{v}\right)+a b\right]+i k \gamma\left(a g_{\nu}+b f_{u}\right)+\gamma^{2}\left(f_{u} g_{v}-f_{\nu} g_{u}\right)$.

The roots of (16) are given by

$$
2 \lambda_{ \pm}=-(\alpha+\mathrm{i} \beta) \pm\left[(\alpha+\mathrm{i} \beta)^{2}-4(\sigma+\mathrm{i} \omega)\right]^{\frac{1}{2}}
$$

where

$$
\begin{aligned}
& \alpha=k^{2}(1+d)-\gamma\left(f_{u}+g_{v}\right), \\
& \beta=-k p, \\
& \sigma=d k^{4}-k^{2}\left[\gamma\left(g_{v}+d f_{u}\right)+\frac{1}{2}\left(p^{2}-q^{2}\right)\right]+\gamma^{2}\left(f_{u} g_{v}-f_{v} g_{u}\right), \\
& \omega=-\frac{1}{2} k^{3}[p(d+1)+q(d-1)]+\frac{1}{2} k \gamma\left[(p+q) g_{v}+(p-q) f_{u}\right] .
\end{aligned}
$$

We may rewrite the solutions (18) in the form

$$
\begin{aligned}
& 2 \operatorname{Re}[\lambda]=-\alpha+\left[\frac{r}{2}\right]^{\frac{1}{2}}\left[1+\frac{1}{\left(1+\phi^{2}\right)^{\frac{1}{2}}}\right]^{\frac{1}{2}}, \\
& 2 \operatorname{Im}[\lambda]=-\sigma+\left[\frac{r}{2}\right]^{\frac{1}{2}}\left[1-\frac{1}{\left(1+\phi^{2}\right)^{\frac{1}{2}}}\right]^{\frac{1}{2}},
\end{aligned}
$$

where

$$
\begin{aligned}
& r=\left[\left(\alpha^{2}-\beta^{2}-4 \sigma\right)^{2}+(2 \alpha \beta-4 \omega)^{2}\right]^{\frac{1}{2}}, \\
& \phi=\frac{2 \alpha \beta-4 \omega}{\alpha^{2}-\beta^{2}-4 \omega} .
\end{aligned}
$$

The steady state $\left(u_{0}, v_{0}\right)$ is linearly stable if $\operatorname{Re}[\lambda]<0$ for all wavenumbers $k$. The conditions for the steady state to be stable in the absence of spatial effects, i.e. $\operatorname{Re}[\lambda(k=0)]<0$, are given by (8) and (9). Given these conditions, the system will become unstable to suitable spatially varying disturbances if 
$\operatorname{Re}[\lambda(k)]>0$ for some $k>0$. These solutions will occur only if $k$ represents an admissible mode, and the implication of this will be discussed in full in a later section. By manipulating (19) using a computer algebra package, it can be shown that if instability does occur for a given (admissible) $k$ then the bifurcation will occur along the line $\operatorname{Re}(\lambda)=0$, that is

$$
\begin{gathered}
d^{3}\left[k^{8}-\gamma f_{u} k^{6}\right]+ \\
d^{2}\left[2 k^{8}+\gamma^{2} k^{4}\left(2 f_{u}^{2}-f_{v} g_{u}+3 f_{u} g_{v}\right)-\gamma k^{6}\left(4 f_{u}+3 g_{v}\right)\right]+ \\
d\left[k^{8}+\gamma^{3} k^{2}\left(-f_{u}^{3}+2 f_{u} f_{v} g_{u}-4 f_{u}^{2} g_{v}+2 f_{u} g_{u} g_{v}-3 f_{u} g_{v}^{2}\right)+\right. \\
\left.\gamma^{2} k^{4}\left(3 f_{u}^{2}-2 f_{v} g_{u}+8 f_{u} g_{v}+3 g_{v}^{2}\right)-\gamma k^{6}\left(+3 f_{u}+4 g_{v}+f_{u} q^{2}\right)+k^{6} q^{3}\right]+ \\
\gamma^{4}\left(f_{u}+g_{v}\right)^{2}(|A|)+\gamma^{3} k^{2}\left(f_{u}+g_{v}\right)\left(2 f_{u} g_{u}-3 f_{u} g_{v}-g_{v}^{2}\right)+ \\
\gamma^{2} k^{4}\left[|A|+2 g_{v}\left(f_{u}+g_{v}\right)\right]-\gamma\left(g_{v} k^{6}\right)+q^{2}\left(g_{v} k^{2} \gamma\right)\left(f_{u} \gamma-k^{2}\right)=0 .
\end{gathered}
$$

This is independent of $p$ and can be solved explicitly for $q$ giving $q^{2}=Q(d ; k)$. We have illustrated this curve in Fig. 1. This gives rise to the third condition for dispersion driven instability, i.e.

$$
q^{2}>Q(d ; k),
$$

for any admissible $k$. In (8) and (9) we have established the conditions for the homogeneous steady state to be stable. If $f_{u}+g_{v}<0$ then either or both of $f_{u}$ and $g_{v}$ is negative. If both $f_{u}$ and $g_{v}$ are negative then (23) implies that $Q(d ; k)<0$ for all $d, k^{2}>0$. Thus $f_{u}$ and $g_{v}$ must have different signs and we assume without loss of generality that $f_{\mathcal{L}}>0$.

There is a critical curve $q^{2}=Q(d ; k)$ dividing stability and instability. If

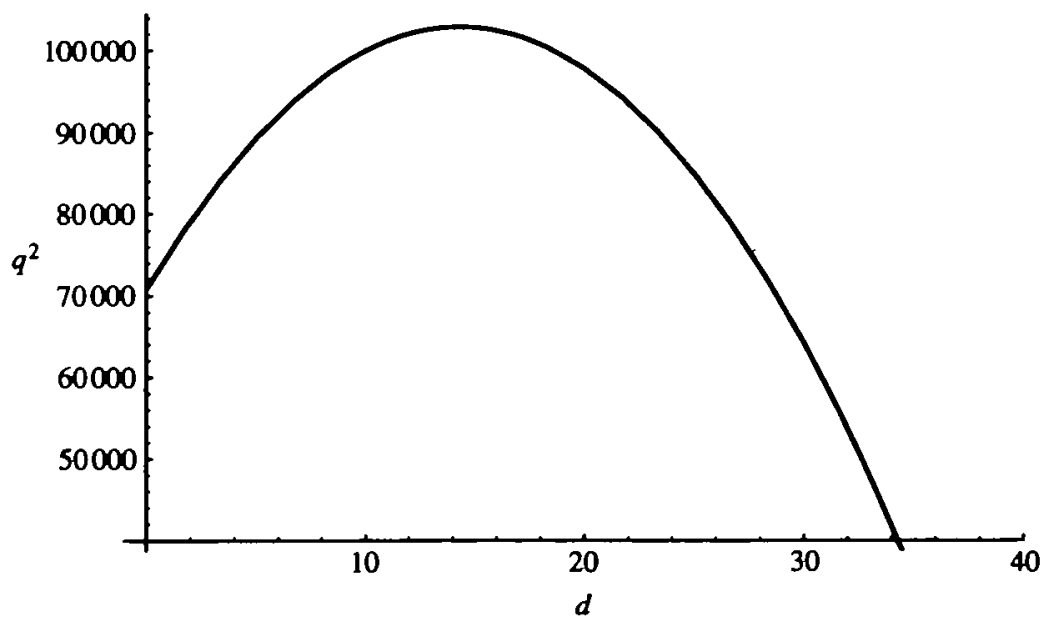

FIG. 1. An example of the curve $Q(d ; k)$ for which $(\partial Q / \partial d)(0 ; k)>0$. Here we are considering a mode 2 solution. Here and in future numerical simulations we use the Schnakenberg (1979) system which is a simple kinetic mechanism of the cross activator-inhibitor type. It can be nondimensionalized to two parameters and is hence convenient to study numerically. In this simulation we have used the Schnakenberg kinetics as defined in (33) and (34) with $A=0.2$ and $B=2 \cdot 0$. The scale factor $\gamma$ is taken as 450 . 
$k^{2}>\gamma f_{u}$, then direct substitution into (23) shows that $Q(d ; k)<0$ for any $d>0$. Hence $Q(d ; k)$ lies below the $d$ axis whenever $k^{2}>\gamma f_{\mu}$, and all unstable wavenumbers satisfy $k^{2}<\gamma f_{\mu}$.

This then implies that the need for autocatalysis and the maximum wavenumber are independent of the presence of advection. We can now use the inequalities (8) and (9) to establish the configurational requirements on the stability matrix. As in the standard case of reaction-diffusion equations without advection there are only two configurations that can satisfy (8) and (9) with $f_{\mu}$ and gu having opposite signs, namely

$$
\left[\begin{array}{ll}
f_{u} & f_{v} \\
g_{u} & g_{\nu}
\end{array}\right] \text { has the sign structure }\left[\begin{array}{ll}
+ & + \\
- & -
\end{array}\right] \text { or }\left[\begin{array}{ll}
+ & - \\
+ & -
\end{array}\right] .
$$

\subsection{Diffusional requirements}

The next issue relates to the ratio of the two diffusion coefficients $d=D_{u} / D_{v}$. In the absence of advection there is a critical diffusion coefficient ratio $d=d_{\mathrm{c}}$ at which the Turing bifurcation occurs. For all values of $d$ below $d_{c}$ the system is stable to small perturbations while for $d>d_{c}$ suitable perturbations grow in time. Here $d_{c}$ is given as the unique root greater than 1 of the quadratic

$$
d_{c}^{2} f_{u}^{2}+2\left(2 f_{v} g_{u}-f_{u} g_{v}\right) d_{c}+g_{v}^{2}=0
$$

(see Murray, 1989). However, in the presence of advection, the corresponding bifurcation occurs as $q^{2}$ increases through $q^{2}=Q(d ; k)$. In some cases $Q(d ; k)$ increases for small $d$ before decreasing to zero for $d=d_{\mathrm{c}}$. This implies two regions of instability with an intervening interval of stability as $d$ is increased for fixed $q$. The derivative of $Q(d ; k)$ at $d=0$ is given by

$$
\left.\frac{\partial Q}{\partial d}\right|_{d=0}=\frac{\gamma\left(f_{u}+g_{\nu}\right)-k^{2}}{g_{\nu}\left(k^{2}-\gamma f_{u}\right)}\left[\left(k^{2}-\gamma f_{u}\right)\left(2 g_{v}^{2}+f_{\nu} g_{u}\right)+\gamma f_{\nu} g_{u} g_{\nu}\right] .
$$

Recalling that $k^{2}<\gamma f_{u}$ along with the kinetic constraints, this implies that:

(1) if $2 g_{v}^{2}+f_{v} g_{u}<0$, then $\partial Q / \partial d$ is strictly positive;

(2) if $2 g_{v}^{2}+f_{v} g_{u}>0$, then the derivative is negative for small wavenumbers and positive for large wavenumbers.

A positive derivative for $Q(d)$ implies that for particular values of $q$, namely $q^{2}>Q(0 ; k)$, a monotonic increase in $d$ will result in instability to perturbations with that wavenumber for small and large $d$, with an interval of stability in between. Thus there are two Turing-type bifurcations as $d$ is varied with $q$ fixed. 


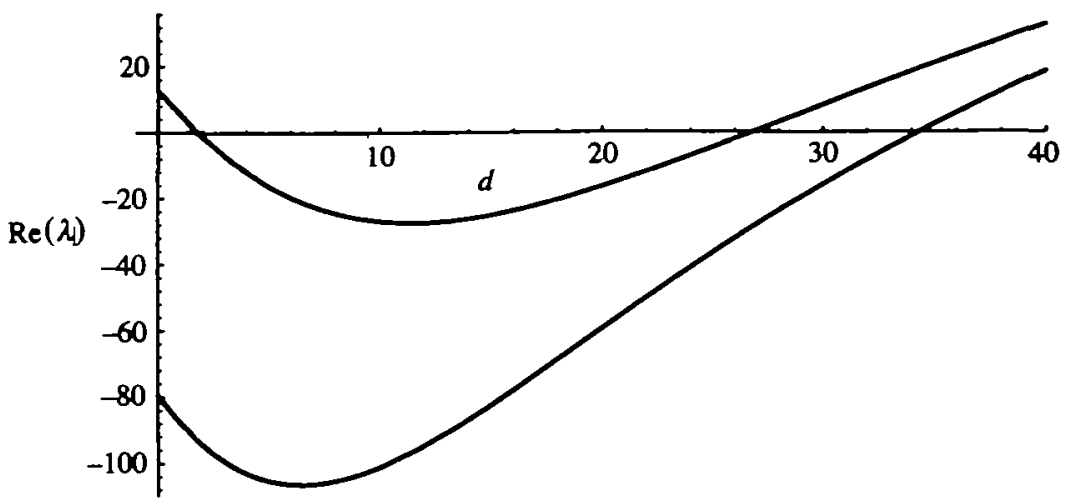

FIG. 2. In the presence of advection for sufficiently large values of $q$, positive growth rates $(\operatorname{Re}(\lambda)>0)$ occur for very small and very large values of $d$ with an intervening area of negative growth rates. In this figure we plot $\operatorname{Re}(\lambda)$ against $d$ as defined in equation (20). For the lower curve $q=\mathbf{2 0 0}$ and there is only one region of positive growth rates for large values of $d$. The upper curve has $q=280$ and shows separated areas of positive growth. In this simulation we have used the Schnakenberg kinetics as defined in (33) and (34) with $A=0.2$ and $B=2 \cdot 0$. The scale factor $\gamma$ is taken as 450 .

In Fig. $2, \operatorname{Re}[\lambda]$ is plotted against $d$ for two different values of $q$ with a particular set of kinetics, and for the larger value of $q$ it is clear that there are two separate Turing bifurcations. Note that the maximum of $Q(d ; k)$ can easily be determined analytically as the positive root of the quadratic $\partial Q / \partial d=0$.

\section{The form of spatial patterns}

\subsection{The dispersion relation}

We are considering solutions of the linearized system with

$$
(u, v) \propto \mathrm{e}^{\lambda(k) t+k x}
$$

and these will grow in time if and only if $\operatorname{Re}(\lambda)>0$. On an infinite domain, the condition $\lambda(k)>0$ for some $k$ is sufficient for the existence of pattern; however, on finite domains, the boundary conditions impose further restrictions. Only certain modes which satisfy the boundary conditions can be accommodated, and if $\lambda(k)$ is not positive for such a wavenumber then no pattern emerges. Our notation henceforth is that a mode $n$ solution has wavenumber $k=2 \pi n$.

\subsection{Initial mode selected}

The curve $q^{2}=Q(d ; k)$, illustrated in Fig. 1 , divides the $q-d$ plane into stable and unstable regions for perturbations of a particular wavenumber $k$. In applications, however, our interest is in whether the steady state $\left(u_{0}, v_{0}\right)$ is stable or unstable to a perturbation of any admissible wavenumber, on a given domain. The curve corresponding to this in the $q-d$ plane is $q^{2}=Q^{*}(d)=\min _{n} Q(d ; k=2 n \pi)$. A 


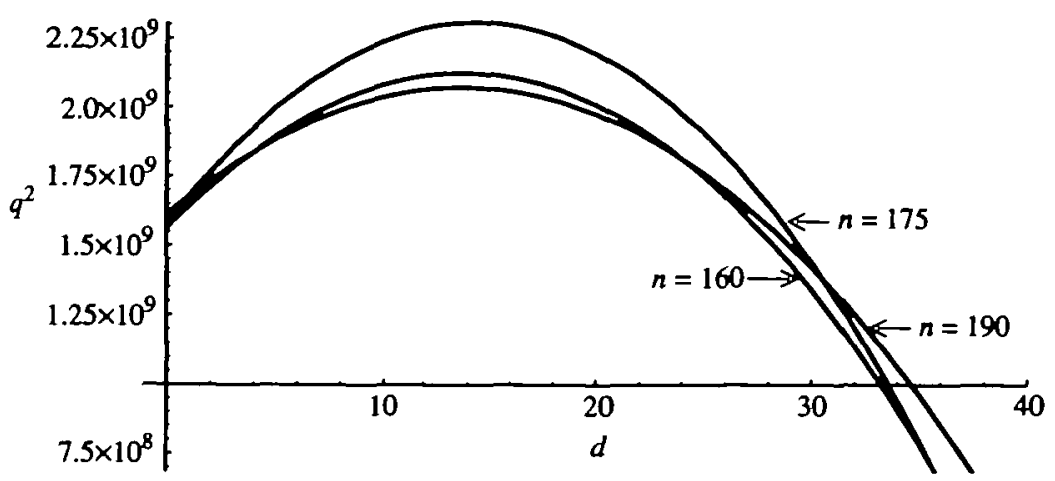

FIG. 3. A typical case illustrates that, for sufficiently large values of the space parameter $\gamma$, the initial mode of bifurcation is not uniform. Here the domain size is much larger than that in Fig. 1 and we have chosen the scale parameter $\gamma=10^{7}$. The three curves drawn from (23) represent the line of bifurcation into patterns with mode numbers mentioned alongside the curves. In this case the primary bifurcation is into different modes in different regions of the $q-d$ plane.

key issue is whether $Q^{*}(d)$ consists simply of a single $Q(d ; 2 \pi n)$ curve or whether it is composed of segments of different $Q(d ; 2 \pi n)$ curves for different values of $n$. Numerical investigation shows that both cases occur and suggests that for small domains the primary bifurcation curve has a single mode, but for large domains the initial bifurcation is into patterns with different mode numbers at different points in the $q-d$ plane; we will confirm this analytically by considering the $q=0$ and $d=0$ cases. Thus the primary bifurcation curve $q^{2}=Q^{*}(d)$ is in general composed of portions of the curves $q^{2}=Q(d ; 2 \pi n)$ for different values of $n$. In Fig. 3 we show a numerical demonstration of a case in which this initial bifurcation curve is composed of three different modes.

A convenient way to study the nonuniformity of the initial mode of bifurcation in large domains is by looking at the end points of the curve described in Fig. 1, that is, the case $q=0$ and $d=0$.

(a) Bifurcation when $q=0$ The point at which the curve intersects the $d$ axis is the point at which the advective terms are the same or are absent. The system then reduces to a standard reaction-diffusion system and the initial bifurcation into instability is through the wavenumber satisfying the quadratic

$$
d k^{4}-\gamma k^{2}\left(d f_{\mu}+g_{v}\right)+\gamma^{2}|A|=0
$$

and the initial mode selected at the primary bifurcation is given by the maximum of the above curve, which is

$$
k_{\mathrm{m}}^{2}=\gamma \frac{d f_{u}+g_{v}}{2 d}
$$

We have illustrated this mode selection for different domain sizes in Fig. 4. 


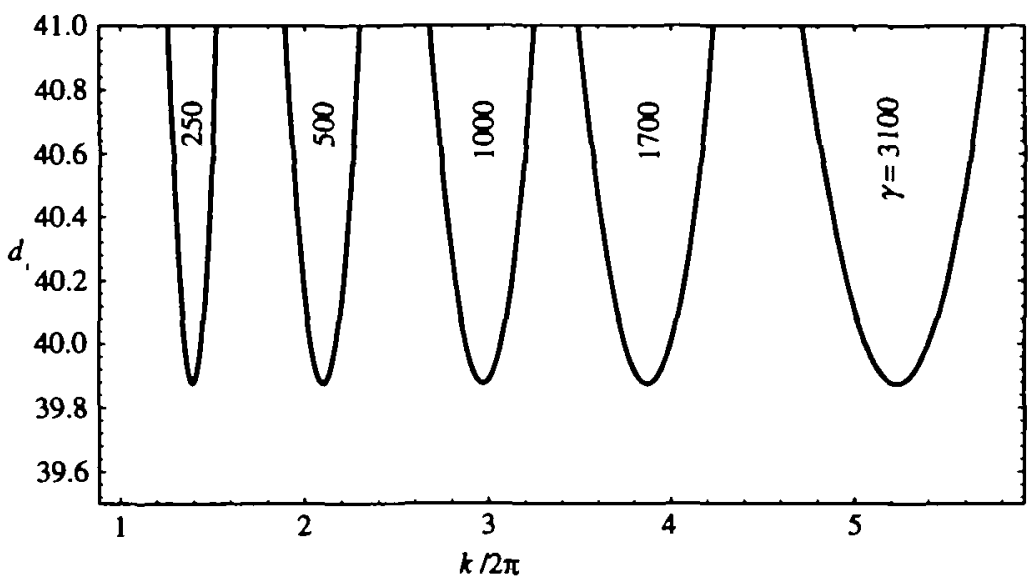

Fig. 4. An illustration of the curve described in (28) for different values of the space parameter $\gamma$. This represents the prediction made by the linear theory for $k / 2 \pi$, where $k$ is the wavenumber of the pattern when the advection terms are either absent or equal for the two morphogens. The kinetics used here are the Schnakenberg kinetics with $A=0.2$ and $B=2 \cdot 0$.

(b) Bifurcation when $d=0$ Another point of interest on the curve given in Fig. 1 is the point at which it intersects the $q^{2}$ axis, $d=0$. Physically this implies a situation where the inhibitor does not diffuse or the activator has a very large (effectively infinite) diffusion coefficient compared to the inhibitor. In the absence of advection the inhibitor needs to diffuse faster than the activator (short-range activation, long-range inhibition) for diffusion-driven instability to occur. However, in the presence of advection, Turing-type bifurcations can occur even when $d=0$, and from (23) the bifurcation at $d=0$ is given by

$$
\begin{aligned}
\gamma^{4}\left(f_{u}+g_{v}\right)^{2}(|A|)+\gamma^{3} k^{2}\left(f_{u}+g_{v}\right)\left(2 f_{v} g_{u}-3 f_{u} g_{v}-g_{v}^{2}\right) \\
+\gamma^{2} k^{4}\left(|A|+2 g_{v}\left(f_{u}+g_{v}\right)\right)-\gamma\left(g_{v} k^{6}\right)+q^{2}\left(g_{v} k^{2} \gamma\right)\left(f_{u} \gamma-k^{2}\right)=0 .
\end{aligned}
$$

Numerical investigation shows that for small domains the primary bifurcation on the $d=0$ and $q^{2}=0$ curves occur for wavenumbers which, though different, are sufficiently close that the nearest admissible wavenumber is the same. However, as the domains become larger, the two wavenumbers move apart. Since we are dealing with a finite domain and the boundary conditions are periodic, the wavenumbers have to be discrete multiples of $2 \pi$. At a certain domain size they are more than $2 \pi$ apart and then the mode numbers of the initial bifurcation on the two areas are no longer the same. We illustrate this mode selection for different domain sizes in Figs 4 and 5. 


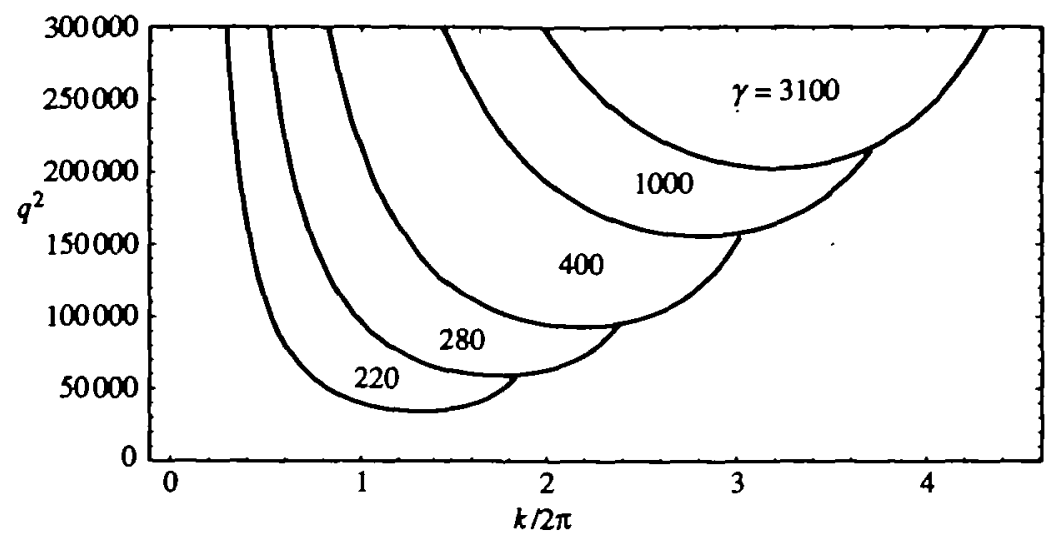

FIG. 5. An illustration of the prediction made by the linear theory of $k / 2 \pi$, where $k$ is the wavenumber, when the inhibitor does not diffuse, i.e. $d=0$. We plot the equation (30) for different domain sizes. Depending on the choice of the domain size reflected in varying values of $\gamma$, the initual mode selected varies. The kinetics are the Schnakenberg kinetics with $A=0.2$ and $B=2.0$.

\section{The nature of the advection terms}

It emerges from the analysis that the parameters $p$ and $q(p=a+b, q=a-b)$ represent a convenient way to study the nature of the advective influence on the features of the model. Both the phase difference and the growth rate $(\operatorname{Re}(\lambda))$ are independent of the parameter $p$ and depend only on $q$. However, $\operatorname{Im}(\lambda)$ depends on both $p$ and $q$. In other words, the phase difference and growth of modes depend only on the difference of the advective terms, but the temporal movement of the solution when a mode is unstable depends on both their sum and difference.

The physical meaning of $\operatorname{Im}(\lambda) \neq 0$ is that the solutions are not stationary in time. The whole pattern of morphogens moves with a uniform 'phase velocity'. Since the boundary conditions are periodic, a convenient way to visualize the solution is as wrapped around a rotating cylinder. The period of rotation of the cylinder is given by $2 \pi / \operatorname{Im}[\lambda]$. Now the parameter $p$ simply corresponds to a rotation in the observer's frame of reference, and if this frame of reference is moving with the same velocity as the angular velocity of the cylinder then the solutions will appear to be stationary. As discussed earlier the phase difference $\Phi$ and the growth rate $\operatorname{Re}(\lambda)$ are independent of $p$ but $\operatorname{Im}(\lambda)$ depends on $p$. So, by an appropriate choice of $p$, we can induce a solution with any particular $\Phi$ and $\operatorname{Re}(\lambda)$ to be stationary. Using (20) we can derive an analytical expression for a $p_{\text {critteal }}$ such that when $p=p_{\text {crtical }}$ the solutions are stationary in time. This is given by

$$
p_{\text {critical }}=\frac{1}{k}\left[\frac{r}{2}\right]^{\frac{1}{2}}\left[1-\frac{1}{\left(1+\phi^{2}\right)^{\frac{1}{2}}}\right]^{\frac{1}{2}},
$$

where $r$ and $\phi$ are given in (21) and (22). Note that this critical value is different for different modes. 


\section{Phase differences}

The fundamental new phenomenon resulting from the inclusion of advection is that the patterns of the two morphogens are spatially out of phase. Since $u$ and $v$ are sinusoidal functions, we can write them in the form $r_{1} \mathrm{e}^{\mathrm{i} \theta_{1}}$ and $r_{2} \mathrm{e}^{\mathrm{i} \theta_{2}}$, respectively, and calculate the phase difference as $\Phi=\theta_{1}-\theta_{2}$. $\Phi$ can be determined analytically from (13) and (14) as

$$
\Phi=\arctan \left[\frac{-a k+\operatorname{Im}(\lambda)}{k^{2}-\gamma f_{u}+\operatorname{Re}(\lambda)}\right]
$$

However, we are using analysis on the linear system to make predictions about the full nonlinear system. These predictions will be valid only close to the primary bifurcations where the effects of the nonlinearities are small. Using expression (23) we can determine $q^{2}(d)=Q(d ; k)$ along the line $\operatorname{Re}(\lambda)=0$, and it is along this line in the $q-d$ plane that we study the phase difference. It is straightforward to determine an analytical expression, albeit algebraically complicated, for the phase difference, and a typical case is illustrated in Fig. 6.

We have been unable to use the equation for the phase difference along the line $\operatorname{Re}(\lambda)=0$ so as to determine the maximum phase difference that occurs. However, our numerical investigations suggest that the maximum phase difference seems to occur at $d=0$ and it is straightforward to determine an analytical expression for this. This maximum phase difference depends on the kinetics and with the Schnakenberg kinetics the maximum is about $60^{\circ}$ for $A=0.2$ and $B=2 \cdot 0$. Another point of interest is that Fig. 6 suggests that the derivative at

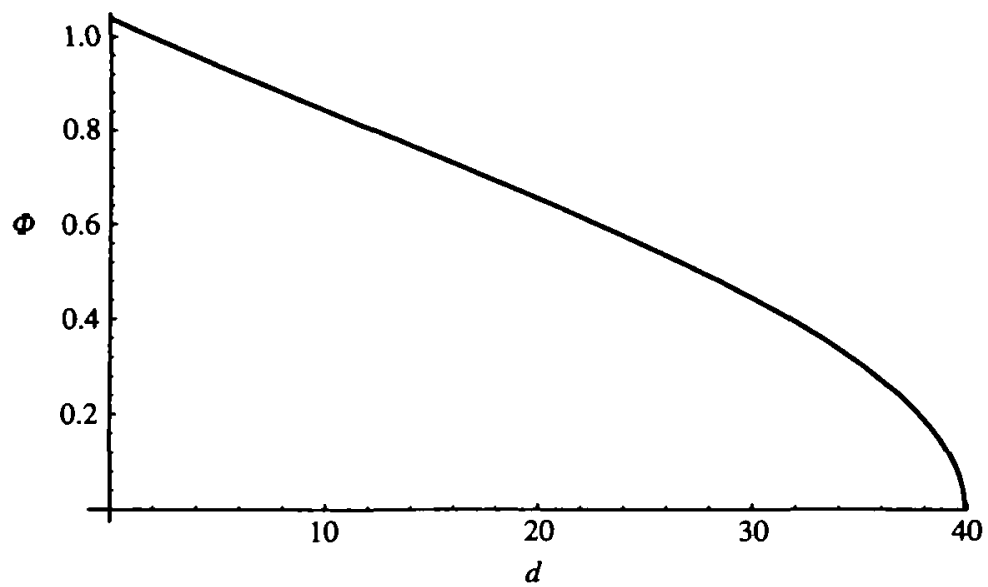

FIG. 6. A plot of the phase difference along the line of bifurcation, i.e. $\operatorname{Re}(\lambda)=0$. We have substituted for $q$ from (23) in (32) to obtain this plot. The parameter values used are $\gamma=450$ and the kinetics are the Schnakenberg kinetics with $A=0.2$ and $B=2 \cdot 0$. Here we are considering a mode 2 solution. 
the zero of $\Phi(d)$ is large (though finite). This is significant in applications, meaning that for small values of $q$ appreciable phase differences are obtained.

\section{Numerical simulations}

We numerically investigated equations (3) and (4) with kinetics proposed by Schnakenberg (1979). With appropriate nondimensionalization in one space dimension, the equations are

$$
\begin{aligned}
& u_{t}=\gamma\left(A-u+u^{2} v\right)+a u_{x}+u_{x x}, \\
& v_{t}=\gamma\left(B+u^{2} v\right)+b v_{x}+d v_{x x},
\end{aligned}
$$

on $0 \leqslant x \leqslant 1$. Here $\gamma$ is the scale factor proportional to the squared length of the domain and $A, B, a, b, d$ are constants. We investigated the equations with boundary conditions (5) and (6) and with initial conditions being small perturbations about the steady state of the kinetics. We solved (33) and (34) using both an explicit finite-difference scheme and an implicit theta scheme. For the explicit scheme we used the stability criteria for the diffusion equation, i.e. $v=$ $d \Delta t /(\Delta x)^{2} \leqslant 0.5$ (see Smith, 1985, for details), where $\Delta x$ is the spatial grid size and $\Delta t$ the time step. When diffusion is predominant, values of $v$ close to 0.5 were adequate. But as advection becomes predominant, smaller values of $v$ are required, typically reaching $0 \cdot 1$ for $d=0$. In Fig. 7 we demonstrate the spatial distribution of $u$ and $v$ at different points along the line of bifurcation in the $q-d$ plane described in (23). A comparison of the analytically predicted phase difference with that computed numerically showed a very close agreement. We were able to confirm that by choosing $p=p_{\text {critical }}$ as described in (31) the solutions are stationary in time. Moreover, we were able to demonstrate numerically the occurrence of a Turing-type bifurcation with $d=0$ in (30) and the occurrence of two Turing-type bifurcations as described in the section on diffusional requirements.

\section{Applications in morphogenesis}

Russell (1985) proposed a model to explain the segmentation pattern seen in Drosophila larvae. In his formulation of this model Russell has not attempted to simulate the behaviour of any specific set of reaction-diffusion equations; rather his discussion is abstract and he assumes chemical waves can be generated in the organism. Thus he supposes that the concentration of the two morphogens $w$ and $v$ vary independently along the embryonic axis in a sinusoidal manner. Specifically he takes $w=m_{1}+n_{1} \sin x$ and $v=m_{2}+n_{2} \sin (x+\phi)$, where $\left|m_{1}-m_{2}\right|$ is the difference in equilibrium and $\phi$ the phase difference between the chemical waves of the two morphogens. The relative concentration of the two morphogens may be thought of as defining a quantity $\alpha$ that varies periodically as a function of $x$ :

$$
\alpha=\arcsin \frac{-w}{\left(w^{2}+v^{2}\right)^{\frac{1}{2}}}
$$


(a)

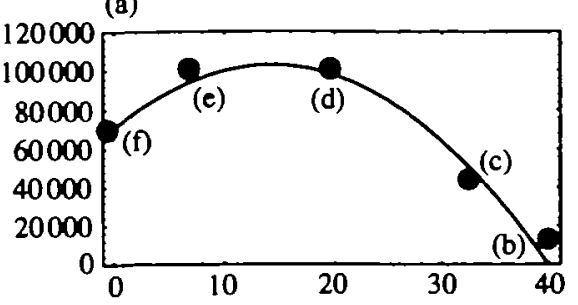

(c)
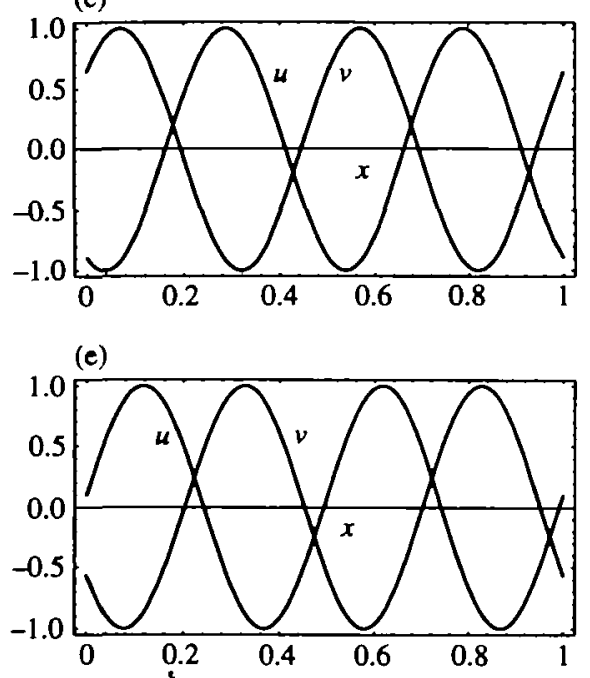

(b)

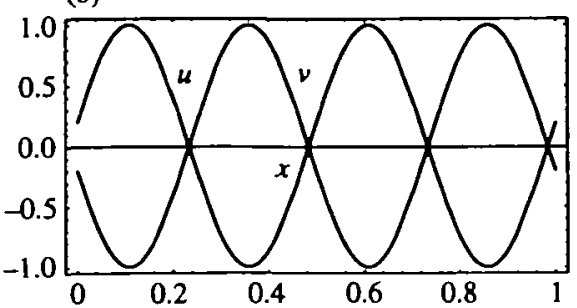

(d)

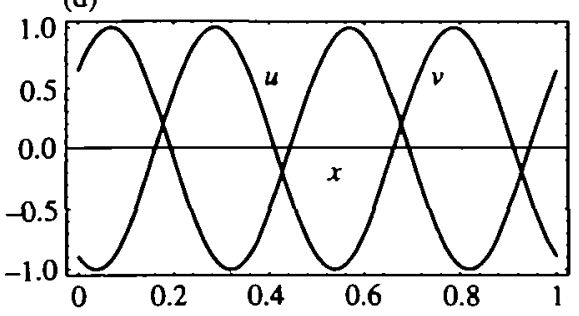

(f)

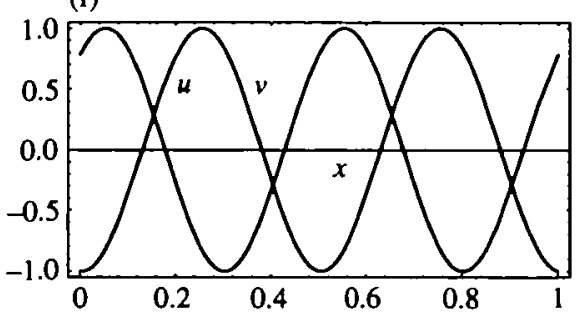

FIG. 7. The behaviour of the nonlinear system described in equations (3) and (4) along the line of bifurcation in the $q-d$ plane. (a) The analytical prediction from the linear system of the bifurcation curve as described in (23). We are looking for a mode 2 solution here which is the sole inttial mode of bifurcation when the space parameter $\gamma=450$. In $(b-f)$ we show the numerical solutions for the five sets of values marked in (a). In (b) the diffusion coefficient $d=40.3$ and the difference of the advection coefficients $q=0$; in (c) $d=32 \cdot 4, q=232$; in (d) $d=20 \cdot 4, q=322$; in (e) $d=8 \cdot 4, q=320$; and in (f) $d=0 \cdot 0, q=270$. As is evident from (f) oscillatory solutions occur even when the inhibitor does not diffuse. Throughout we have used the Schnakenberg kinetics with $A=0.2$ and $B=2 \cdot 0$.

This idea is illustrated in Fig. 8. In the wild type (i.e. normal larvae) the phase difference is set to $\pi / 2$ and $m_{1}=m_{2}=0, n_{1}=n_{2}=1$. Morphological variations in the Drosophila larva are very well documented (see Nusslein-Volhard \& Wieschaus, 1980, for a review). Segment polarity mutations and growth contraction/expansion are common variations. In the segment polarity mutants in each segment a defined fraction of the normal pattern is deleted and the remainder is present as a mirror image duplication. Russell (1985) proposes that these variations can be explained on the basis of the patterning variable $\alpha$, given changes in the equilibrium and phase differences of the morphogen patterns (see Fig. 8).

We suggest that with $w=\partial u / \partial x$ and $(a, b)=(0,0)(33)$ and (34) can be used to 

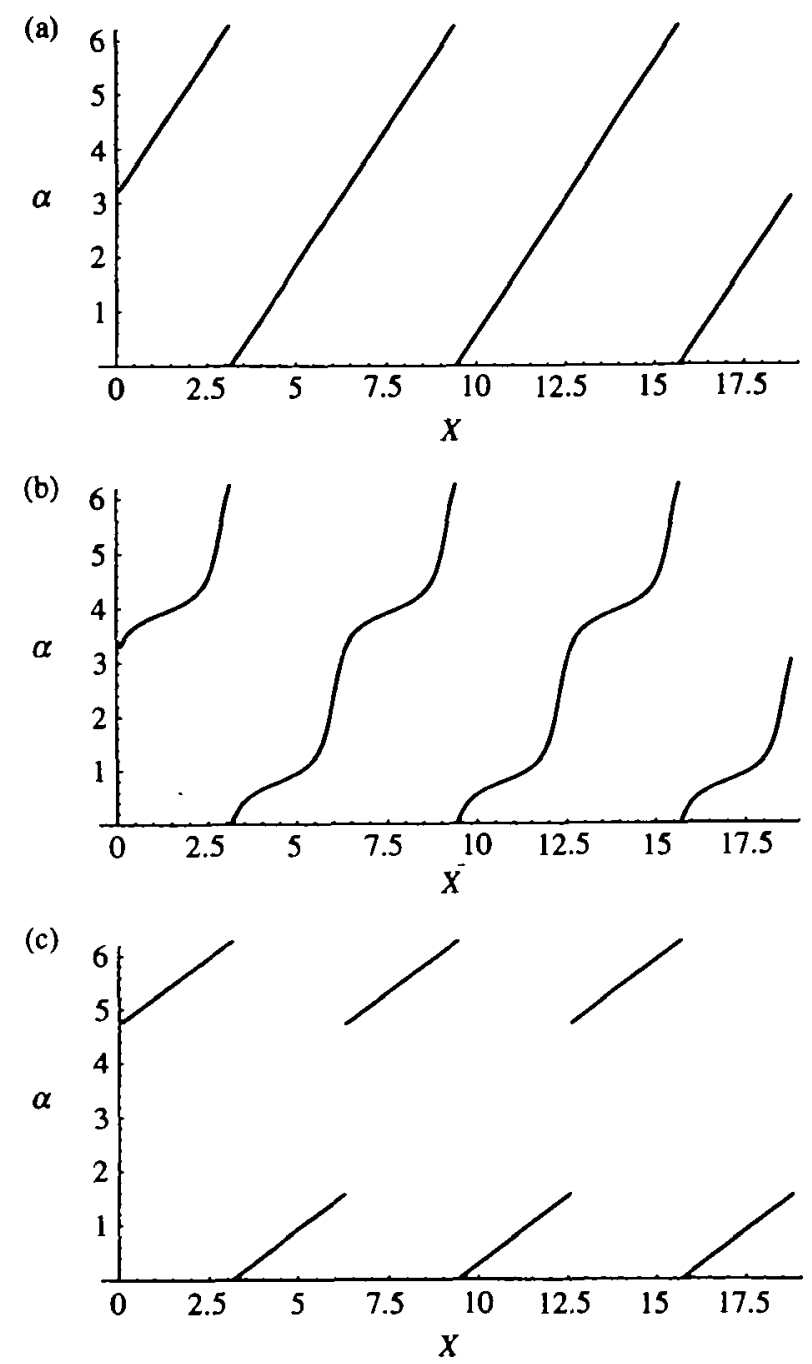

Fig. 8. The distribution of the relative concentration of the two morphogens as a function of space described in (35) with $v$ and $w$ as given in the text. In all cases $n 1=n 2=1$. (a) The occurrence of reiterated linear gradients of $\alpha$; here $\phi=\pi / 2, m_{1}=m_{2}=0$. (b) The simulation for growth contraction and expansion arising from a change in the phase difference; here $\phi=\pi / 6$. (c) The simulation for segment polarity mutants; here $\phi=\pi / 2, m_{1}=0$, and $m_{2}=-1 \cdot 0$.

model the wild type of the Drosophila larva. In Table 1 we list some parameter values which give the appropriate phase and equilibrium differences to model segment polarity mutants and growth contraction and expansion. However, it is important to stress that there is currently no experimental evidence for advection being a significant phenomenon in the Drosophila larva. The key implications of this work are that advection does represent a natural way in which phase differences and other novel phenomena could arise in diffusion-driven patterns. 


\section{TABLE 1}

A sampling of the ualues which can give rise to the differences in equilibrium and phase of the two chemical waves to simulate some of the morphological patterns seen in the Drosophila larva. $A$ and $B$ are the parameters from the kinetics of the Schnakenberg system described in (33) and (34), $d$ is the diffusion coefficient of the inhibitor and $q=a-b$ is the difference of the advection coefficients. The scale parameter $\gamma=450$

\begin{tabular}{lcccc}
\hline \multirow{1}{*}{$\begin{array}{c}\text { Morphological } \\
\text { pattern }\end{array}$} & \multicolumn{2}{c}{ Kinetics } & & Diffusion \\
\cline { 2 - 4 } & $A$ & $B$ & $d$ & $\begin{array}{c}\text { Advection } \\
q\end{array}$ \\
\hline Normal Drosophila larvae & 0.2 & 2.0 & 39.9 & 0.0 \\
Growth contraction \& expansion & 0.2 & 2.0 & 8.4 & 320.0 \\
Segment polarity mutants & 0.1 & 0.9 & 8.56 & 0.0 \\
\hline
\end{tabular}

\section{Acknowledgement}

AJP wishes to thank the Radhakrishnan Scholarship, which funds his research.

\section{REFERENCES}

Benson, D. L., Sherratt, J. A., \& Main, P. K., 1993. Diffusion driven instability in an inhomogeneous domain. Bull. Math. Biol. 55, 365-84.

Casten, R. G., \& Holland, C. J., 1977. Stability properties of solutions to systems of reaction-diffusion equations. SIAM J. Appl. Math. 33, 353-64.

Dlllon, R., Maini, P. K., \& Othmer, H. G., 1994. Pattern formation in generalised Turing systems. J. Math. Biol. 32, 345.

Meinhardt, H., 1982. Models of Biological Pattern Formation. London: Academic Press.

Murray, J. D., 1989. Mathematical Biology. Berlin: Springer.

Nusslein-Volmard, C., \& Wieschaus, E., 1980. Mutations affecting segment number and polarity in Drosophila. Nature $\mathbf{2 8 7 , 7 9 5 - 8 0 1 . ~}$

Russell, M. A., 1985. Postional information in insect segments. Dev. Biol. 108, 269-83.

SCHNAKENBERo, J., 1979. Simple chemical reaction systems with limit cycle behaviour. $J$. Theor. Biol. 81, 389-400.

SMrTH, G. D., 1985. Numerical Solutions of Partial Differential Equations: Finite Difference Methods. Oxford: Clarendon Press.

Turing, A. M. 1952. The chemical basis of morphogenesis. Phil. Trans. R. Soc. Lond. B 237, 31-72. Reprinted in Bull. Math. Biol. 52, 153-97, 1990. 